\title{
THE PHYSIOLOGY OF STEPPING.*
}

\author{
By T. GRAHAM BROWN, Cardiff.
}

\section{I.-THE PRODUCTION OF RHYTHM.}

The writer ${ }^{1}$ has shown that the rhythmic alternation of movement in such acts as stepping is not produced by the action of rhythmically-timed stimuli evoked in the moving limbs themselves. The rhythm is essentially produced in the nervous centres. He also showed that rhythmic movements may occur when two continuous movements (flexion and extension) antagonize one another. It would appear that the rhythm arises under the physiological interference' of the two arrhythmic activities. The essential condition of stepping is the production of two antagonistic activities (flexion and extension) of continuous type and of more or less equal intensities in the spinal centres.

Other workers have accepted this view. They have, however, translated it to mean that the rhythm arises under equality of the excitation and inhibition evoked in each group of motor neurones. But the inhibition and excitation given by the same stimulus are not equal; and the inhibition given by extension-producing stimuli is less nearly equal to the excitation than is the inhibition given by flexion-producing stimuli to the excitation given by them.

Exact equality may, of course, occur between the excitation given by one stimulus and the inhibition given by another in one group of motor neurones (e.g., flexor), provided the different intensities of the two stimuli are appropriate. But there will then be inequality of excitation and inhibition (from antagonistic stimuli) acting upon the other group of motor neurones. (This follows algebraically from the fact of inhibition being less than the excitation given by at least one of the two stimuli.) In other words, when exact equality occurs between the excitation and inhibition playing upon one group of neurones (e.g., flexor), the inhibition must be less than the excitation playing upon the other (e.g., extensor), and that other group must have a discharge greater than normal. Therefore the two antagonistic groups of motor neurones cannot discharge rhythmically at one and the same time if exact equality between the inhibition and excitation playing upon either is the condition of its 
rhythm. Or, if the condition is merely a close approximation to equality, the rhythmic discharge will be best marked in that muscle the motor neurones of which are most equally acted upon by excitation and inhibition.

Simultaneous rhythmic movement in two antagonistic muscles is, however, one of the chief features of stepping (and so on); and I have found that if the excitation and inhibition are nearly equal in one group of motor neurones, the rhythm is best marked in the other muscle. Therefore, the condition of rhythmic discharge is not equality between the inhibition and excitation playing upon each group of motor neurones.

We are thus faced with the question, What is the condition of the rhythmic discharge in such coarse rhythms as stepping?

If the excitation which plays upon a group of motor neurones is greater than a simultaneous inhibition which acts upon it, there will be an overplus of excitation after the inhibition has had its reducing effect. This may be termed the 'resultant excitation', and the discharge which is thereby conditioned may be termed the 'resultant discharge'. This state occurs, of course, in compound reflexes. For instance, if strong flexion is antagonized by moderate extension, there is a resultant discharge in the group of flexor motor neurones; for the flexion-reflex wins although it may be reduced in the combat.

We obtain an 'intensity series' of compound reactions if we take 'simple' extensions of constant intensity and antagonize them with different intensities of simple flexion. Suppose that the series starts at the extreme of minimal flexion; there is then resultant discharge in the extensor group of neurones during the compound reflex, and none in the flexor. As the intensity of simple flexion is raised in succeeding reactions, the extensor resultant discharge falls (because the inhibition factor rises); then a resultant discharge appears in the flexor group of motor neurones, at a certain intensity of 'simple' flexion, and goes on increasing with further increase in the intensity of flexion.

Therefore one resultant discharge (extensor) progressively falls from a high value to zero, and the other (flexor) begins to rise from zero before the former has fallen absolutely to zero (for there is simultaneous discharge of the two groups in the middle of the series). There must be an intermediate point at which the two resultant discharges are equal. (I measure resultant discharge-flexor or extensor - as the ratio of compound muscle contraction to the contraction in the maximal simple flexion- or extension-reflex.)

My results indicate that it is at this point (and round about it) that rhythmic discharges occur. The essential condition of rhythmic 
discharge (stepping, and so on) is approximate equality between the resultant discharges of two antagonistic groups of motor neurones. The rhythmic movements are of greatest magnitude and most 'complete' in type where the two resultant discharges are of the greatest intensity whilst remaining equal. It looks almost as if the two antagonistic groups of motor neurones (e.g., flexor and extensor) may perhaps 'interfere' with one another to give the rhythmic discharge in both. But the conditions of any such interaction can at present only be surmised.

\section{II.-THE CONDITIONS OF BILATERAL AND UNILATERAL STEPPING.}

Sherrington's ${ }^{2}$ theory of the mechanism of stepping and hopping at present holds the field. But it is founded on the assumption that the excitation and inhibition given by the same stimulus are equal. As this is not so, the theory must be reconsidered.

He starts by making the observations that a strong flexion can completely knock out a maximal extension, but that a weak extension knocks out a weak flexion. He measures the intensities of the reflexes by the electric strengths of the stimuli used, and he comes to the conclusion that the intensity gradient of the extension-reflex is less steep than that of the flexion-reflex to electrical stimuli of the same values. This conclusion is not drawn directly from observation of the actual intensity series of reflexes, but from the observations described above; and it is also reinforced by the assumptions that the electric stimulus is a true measure of the reflex it evokes and that the inhibition and excitation given by a stimulus are equal.

I have directly measured the intensity series of the two reflexes, and it appears that the exact opposite takes place in good preparations of the 'decerebrate' cat (cat with the brain removed from above the level of the anterior colliculi).

Stimulation of one and the same afferent nerve in a limb gives flexion in the same limb and extension in the crossed limb of the pair. If the stimuli are progressively increased in intensity from threshold up, the crossed extension rises at a steeper gradient than the same-sided flexion. It attains its maximum while the same-sided flexion is still moderate, or even weak. Further increase in the intensity of stimulation gives further increase in the intensity of flexion while the extension merely remains maximal (or perhaps actually diminishes a little).

The results from which Sherrington assumed the lesser gradient of the extension-reflex were obtained by the application of equal intensities of stimuli in ascending series to two antagonistic afferent nerves 
(right and left). He thus compounded flexion and extension in either limb. After the first few stimuli he was probably compounding maximal extension with sub-maximal degrees of flexion in both limbs. If this was the case; it is easy to see that the increasing flexions against constant extensions gave ever greater flexor contraction and extensor relaxation (resultant flexion) in both limbs. The inhibition given by the constant extension would then appear to be relatively smaller with each increase in intensity of the flexion against which it acted. This was the fact from which the lesser gradient of extension was assumed; it is more easily explained as due to the observed steeper gradient of extension.

My view of the matter is strongly supported by the measurement of the intensity of extension which (when antagonized by flexion) gives the best stepping. I find that the extension-reflex is then usually maximal (or nearly so). Sherrington's statement is that the extension stimulus should be mild or moderate. But he measures the extension stimulus by the intensity of the same-sided flexion reflex which is also evoked by it; and I have shown that a 'weak' stimulus* gives a maximal extension reflex (because of the greater intensity gradient of extension). Therefore the two observations do not clash.

The fact that stepping is obtained where the extension is maximal gives opportunity of a satisfactory explanation of the variations of rhythmic limb movement-galloping, trotting, and stepping in both limbs of a pair at the same time, and in one limb alone while the other is held flexed, and so on.

Maximal extension is induced in the crossed limb (e.g., Left) at every intensity over a very wide range of flexion induced in the same-sided limb ( $\mathbf{R})$. That maximal extension $(\mathrm{L})$ may be antagonized by any of a wide range of intensities of flexion in its own limb $(\mathrm{L})$, and each of these flexions is accompanied by maximal extension in the first limb (R). Thus any combination of flexion (within that wide range) with maximal extension in one limb may be accompanied by the same or any other combination of flexion and maximal extension in the other.

The form of the rhythmic movements depends upon the degree of approximation to equality of the resultant discharges in the antagonistic groups of motor neurones (flexor and extensor) in the same limb. Thus it is possible (on my theory) for any form of rhythmic movement (with certain reservations) in one limb to be accompanied by the same or any other form in the other limb. The theory also shows how the stepping may occur in one limb alone

\footnotetext{
* That is, a stimulus which is 'weak' in its same-sided flexion effect.
} 
while the other is flexed (hopping and the scratch-reflex), or while it is extended (unilateral scraping, as seen in a dog at a rabbit's burrow). The first of these extremes occurs where the same-sided flexion is intense enough to overcome the extension in its own limb, and gives a maximal extension which is antagonized by moderate flexion in the other; and the second occurs where the same-sided flexion is weak enough to be overcome by the maximal extension in its own limb, but yet gives a maximal extension which antagonizes a moderate flexion in the opposite limb.

\section{REFERENCES.}

1 Brown, Graham, Proc. Roy. Soc., B, 1911, Ixxxiv, 308.

2 Sherrington, Ibid., 1913, lxxxvi, 233. 